\title{
Transient headache and neurological deficits with cerebrospinal fluid lymphocytosis (Handl Syndrome): A case report
}

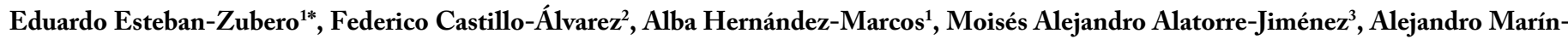 \\ Medina $^{4}$, Amaia Erdocia-Goñi ${ }^{2}$, Carmen Iglesias Gutiérrez-Cecchini ${ }^{2}$ and Noelia Reurich-Gómez ${ }^{2}$ \\ ${ }^{1}$ Emergency Department, Hospital San Pedro, La Rioja, Spain \\ ${ }^{2}$ Department of Neurology, Hospital San Pedro, La Rioja, Spain \\ ${ }^{3}$ Department of Neurosciences, Western biomedical research center, Mexican Institute of Social Security, Guadalajara, Mexico \\ ${ }^{4}$ Department of Genetics, Western biomedical research center, Mexican Institute of Social Security, Guadalajara, Mexico
}

\begin{abstract}
The syndrome of headache with neurologic deficits and cerebrospinal fluid lymphocytosis (HaNDL) is a rare entity. This disease has been related with migrainous headaches. It is a benign disorder, self-limited, and characterized by fluctuating neurological symptoms and cerebrospinal fluid (CSF) lymphocytosis. A small proportion of patients develop intracranial hypertension as a consequence of the illness. Confusional state may be also observed.

We describe a case of a 29 years old man with acute onset of headache, dysthermia sensation, vomits, aphasia, and difficulty to perform daily activities such as unlocking the mobile phone. Blood tests as well as computed tomography (CT) and magnetic resonance imaging (MRI) were normal. Cerebroespinal fluid analysis revealed a lymphocytic pleocytosis (47 cells/ $\mu 1,100 \%$ lymphocytes). Electroencephalogram showed moderate slow rhythm in the left hemisphere, with frontotemporal predominance, and without epileptiform activity. With the diagnosis of HaNDL syndrome the patient was accepted in the Department of Neurology and discharged asymptomatic without treatment.
\end{abstract}

\section{Introduction}

The syndrome of headache with neurologic deficits and cerebrospinal fluid lymphocytosis (HaNDL) is a rare entity [1]. The first description of this disease in the literature was provided in 1981 [2]. The International Headache Society sets forth specific diagnostic criteria that define this syndrome (Table 1) [3].

HaNDL is most frequently observed during the third and fourth decades of life, with no consistent gender predominance identified [4-6]. The etiopathogenesis of HaNDL is poorly understood. Some patients report a preceding non-specific viral prodromal illness [6], but studies have failed to provide a definite viral association [2,3].

Neurologic deficits typically last 15-120 minutes, although a range of 5 minutes to 3 days has been reported. While approximately $75 \%$ of HaNDL cases have a monophasic course, repeated attacks that occur for weeks or even months following the initial attack have been reported. When repeated attacks are observed, neurological deficits may vary due to the involve of different brain regions $[2,3,5]$.

This syndrome is a benign disorder which may be presented as hemi-sensory disturbances (70\%), aphasia (66\%), nausea/vomiting (54\%), weakness (42\%), visual complaints (including decreased vision, homonymous hemianopsia (18\%), photopsia/photophobia (16\%), and diffuse symptoms such as an acute confusional state are including into the neurological manifestations [2,5-7]. However, HaNDL may be accompanied by elevated intracranial pressure (ICP) and papilledema [1], and cerebral herniation [8], which worsen de prognosis.
Herein, we describe a case of HaNDL syndromewith a short review of the literature of this illnes and cases reported.

\section{Case report}

A previously healthy 29 -year-old man with no prior significant medical history was admitted in our Emergency Department reporting acute onset of headache with associated nausea, vomiting, dysthermia sensation, aphasia, and difficulty to perform daily activities such as unlocking the mobile phone. Neurological exploration revealed repetitive language and aphasia without disturbs of cranial nerves. Sensitivity and strenght were symmetrical were preserved. Bilateral flexor cutaneous-plantar réflex as well as visual field test were normal. Any signs of meningism were observed.

During his admission, a blood test was performed revealing only discrete leukocytosis with left deviation $(13.3 \times 1000 / \mu \mathrm{L}$ with $75.5 \%$ neutrophilia). Computed tomography (CT) scan and magnetic resonance imaging (MRI) did not show any disturbs (Figure 1). Electroencephalogram (EEG) showed moderate slow rhythm in the left hemisphere, with frontotemporal predominance, and without

Correspondence to: Eduardo Esteban Zubero, Emergency Department of Hospital San Pedro-26006, Logroño, Spain, Tel: +34654123994; Email: eezubero@gmail.com

Key words: headache, migraine, lymphocytosis

Received: June 02, 2017; Accepted: June 26, 2017; Published: June 28, 2017 


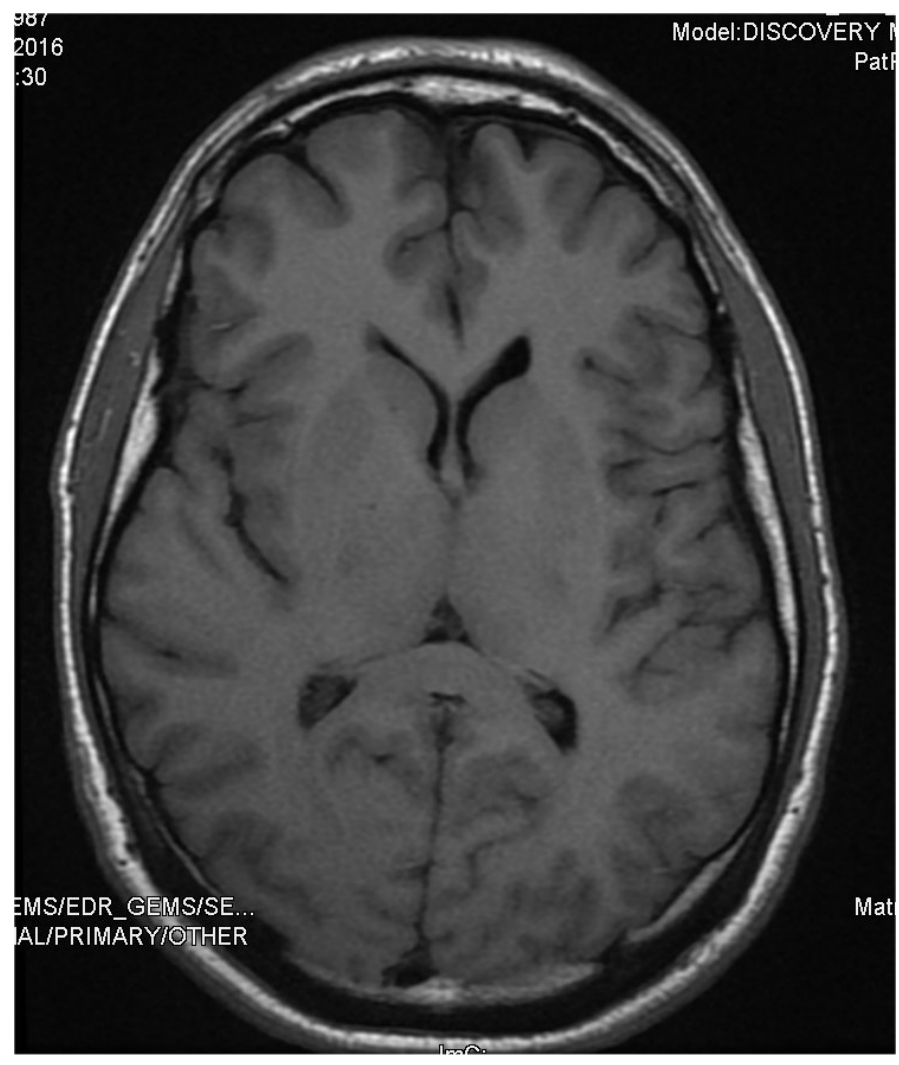

Figure 1. Magnetic resonance imaging (MRI) showing any disturbs.

epileptiform activity. A lumbar puncture was performed to complete the study. Cerebroespinal fluid (CSF) analysis revealed a lymphocytic pleocytosis (47 cells/ $\mu \mathrm{l}, 100 \%$ lymphocytes).

With the diagnosis of HaNDL syndrome the patient was accepted in the Department of Neurology. During his admission, the patient progressed favorably recovering the neurological functionality completely without any specifical treatment. The patient was discharged after 7 days.

\section{Discussion}

The syndrome of headache with neurologic déficits and cerebrospinal fluid lymphocytosis (HaNDL) is a rare entity comprising sudden onset migrainous headaches (generally in headache-naïve individuals) accompanied by a constellation of fluctuating neurological symptoms and CSF lymphocytosis. Neurological symptoms include hemiparesis and hemisensory deficits (which often migrate in a fashion consistent with a cortical spreading depression), aphasia (HANDL favours the left hemisphere), nausea, vomiting, visual disturbance (including decreased vision, homonymous hemianopsia, and photopsia/photophobia), and less commonly, acute confusional state $[9,10]$, elevated intracranial pressure (ICP) and papilledema [1], and cerebral herniation [8], which worsen de prognosis.

The headache, observed frequently after the neurological symptomatology, may be hemicranial or holocranial, pulsatile, and moderate-severe intensity. Its duration is variable, although it is usual to self-limit in few hours [11]. Neurologic deficits typically last 15-120 minutes, although a range of 5 minutes to 3 days has been reported. While approximately $75 \%$ of HaNDL cases have a monophasic course, repeated attacks that occur for weeks or even months following the initial attack have been reported. When repeated attacks are observed, neurological deficits may vary due to the involve of different brain regions $[2,3,5]$.

HaNDL is most frequently observed during the third and fourth decades of life, with no consistent gender predominance identified $[3,5,6]$. However, some case reports are observed in adolescents and childhood [12,13]. The etiopathogenesis of HaNDL is poorly understood. Some patients report a preceding non-specific viral prodromal illness $[6,14]$, but studies have failed to provide a definite viral association $[2,3]$. The presence of antibodies directed against the CACNA1H subunit of the T-type voltage-gated calcium channel in one small study supported the inflammatory or autoimmune hypothesis [15]. Cortical spreading depression-associated cerebral oligaemia has been observed during HaNDL attack, and may play a significant role $[8,16]$.

Diagnosis of HaNDL syndrome is confirmed after having ruled out other diseases of the central nervous system according to the diagnostic criteria showed in Table 1 . CSF pressure and protein are generally elevated at the time of diagnosis, and neuroimaging is usually normal. EEG during the acute symptoms may show slowed rhythm, like our case $[11,14]$. Some authors suggested to perform a CT angiography in patients affected of HaNDL syndrome, neuroimaging tests normal, and suspected diagnosis of vasculitis $[8,11,16]$.

Prognosis of these patients is benign and medical treatment is only necessary to control headache. However, when ICP and papilledema are observed, CSF drainage and acetazolamide trearment are recquired $[8,9,17]$.

In conclussion, HaNDL, a rare disease with diverse clinical manifestations, remains a diagnosis of exclusion. It may be explained on the basis of migrainous or post-viral/inflammatory pathophysiology. The prognosis of this illness is benign and the treatment is symptomatic. However, symptoms including elevated IPC, papilledema and brain herniation may be observed, recquering urgent treatment. We describe a HaNDL case trying to improve the knowledge of this pathology.

\section{Conflicts of interest}

No conflict of interest shown in the realization of this writing.

\section{Funding}

No external funding sources and any comments are received.

Table 1. Diagnostic criteria of the syndrome of transient headache and Neurologic deficits with cerebrospinal fluid lymphocytosis, according to the International Headache Society [4].

\section{A. Episodes of migraine-like headache fulfilling criteria $B$ and $C$}

B. Both of the following:

- Accompanied or shortly preceded by the onset of at least one of the following transient neurological deficits lasting $>4$ hours

a. Hemiparesthesia

b. Dysphagia

c. Hemiparesis

- Associated with cerebrospinal fluid (CSF) lymphocytic pleocytosis ( $>15$ white cells/ $\mathrm{mL}$ ) with negative etiological studies

C. Evidence of causation demonstrated by either or both of the following:

- Headache and transient neurological deficits have developed or significantly worsened in temporal relation to the CSF lymphocytic pleocytosis or led to its discovery

- Headache and transient neurological deficits have significantly improved in parallel with improvements in the CSF lymphocytic pleocytosis

- Not better accounted by another International Classification of Headache Disorders, 3rd edition diagnosis 


\section{References}

1. Morrison DG, Phuyah HK, Reddy AT, Dure LS, Kline LB (2003) Ophthalmologic involvement in the syndrome of headache, neurologic deficits, and cerebrospinal fluid lymphocytosis. Ophthalmology 110:115-118.

2. Bartleson JD, Swanson JW, Whisnant JP (1981) A migrainous syndrome with cerebrospinal fluid pleocytosis. Neurology 31: 1257-1262. [Crossref]

3. Berg MJ, Williams LS (1995) The transient syndrome of headache with neurologic deficits and CSF lymphocytosis. Neurology 45: 1648-1654. [Crossref]

4. Headache Classification Committee of the International Headache Society (IHS) (2013) The International Classification of Headache Disorders, 3rd edition (beta version). Cephalalgia 33: 629-808. [Crossref]

5. Gómez-Aranda F, Cañadillas F, Martí-Massó JF, Díez-Tejedor E, Serrano PJ, et al (1997) Pseudomigraine with temporary neurological symptoms and lymphocytic pleocytosis. A report of 50 cases Brain 120:1105-1113.

6. Filina T, Feja KN, Tolan RW Jr (2013) An adolescent with pseudomigraine, transient headache, neurological deficits, and lymphocytic pleocytosis (HaNDL Syndrome): case report and review of the literature. Clin Pediatr (Phila) 52: 496-502. [Crossref]

7. Mateo I, Pinedo A, Gómez-Beldarrain M, García-Moncó JC (2004) Acute confusional state secondary to transient headache and neurological deficits with cerebrospinal fluid lymphocytosis. Neurologia 19: 763-765.

8. Babi MA, Applebee A, Shapiro R (2017) Syndrome of transient headache and neurologic deficits with cerebrospinal fluid lymphocytosis presenting as acute neurological emergencies. Cephalalgia 37: 284-289. [Crossref]

9. Mulroy E, Yap J, Danesh-Meyer H, Anderson N (2017) Symptomatic intracrania hypertension during recovery from the syndrome of headache with neurologic deficits and cerebrospinal fluid lymphocytosis (HANDL). Pract Neurol 17: 145-148. [Crossref]
10. Lo Re M, di Sapio A, Malentacchi M, Granieri L, Bertolotto A (2015) Acute confusional state in HaNDL syndrome (transient headache and neurologic deficits with cerebrospinal fluid lymphocytosis) Neurol Sci 36: 477-478.

11. Martín-Cascón M, Villaverde-González R, Espinosa-Parra FJ, Meseguer-Noguera R (2015) Syndrome of transient headache and neurologic deficits with cerebrospinal fluid lymphocytosis (HaNDL). Rev Clin Esp 215: 246-247.

12. Rivero-Sanz E, Pias-Peleteiro L, Gonzalez-Alvarez V (2016) HaNDL syndrome in a 14-year-old girl. BMJ Case Rep 2016. [Crossref]

13. Armiento R, Kornberg AJ (2016) Altered conscious state as a presentation of the syndrome of transient headache and neurological deficits with cerebrospinal fluid lymphocytosis (HaNDL syndrome) in a paediatric patient. J Paediatr Child Health 52: 774-776.

14. Verentzioti A, Tavernarakis A, Mamali M, Siatouni A, Gatzonis S (2017) Pseudomigraine with transient neurological deficits and cerebrospinal fluid lymphocytosis or HaNDL syndrome: A case report with confusion and positive IgM antibodies to CMV in serum. Cephalalgia 37: 99-100.

15. Kürtüncü M, Kaya D, Zuliani L, Erdag E, Içöz S, et al. (2013) CACNA1H antibodies associated with headache with neurological deficits and cerebrospinal fluid lymphocytosis (HaNDL). Cephalalgia 33: 123-129.

16. Burke MJ, Lamb MJ, Hohol M, Lay C (2017) Unique CT Perfusion Imaging in a Case of HaNDL: New Insight into HaNDL Pathophysiology and Vasomotor Principles of Cortical Spreading Depression. Headache 57: 129-34.

17. Chan JW, Cheng C (2010) Complete external ophthalmoplegia in headache, neurologic deficits, and cerebrospinal fluid lymphocytosis (HaNDL) syndrome. Eye 24: 198-199.

Copyright: $(2017$ Esteban-Zubero E. This is an open-access article distributed under the terms of the Creative Commons Attribution License, which permits unrestricted use, distribution, and reproduction in any medium, provided the original author and source are credited. 